\title{
Four Different Apparent Diffusion Coefficient Measurement Methods in Breast Masses
}

\author{
Betul Duran and Burcin Agridag Ucpinar \\ Department of Radiology, Sisli Hamidiye Etfal Training and Research Hospital, Istanbul, Turkey
}

\begin{abstract}
Objective: To compare the effect of different quantification methods of ADC values in the evaluation of breast lesions and compare contralateral normal breast tissue ADC value by calculating ADC ratios.

Study Design: Descriptive study.

Place and Duration of the Study: Sisli Etfal Training and Research Hospital, Turkey, between February 2019 and December 2020.

Methodology: Two hundred and forty-six breast MRI scans with DWI of the patients with biopsy proven unilateral breast lesions were studied. ADC measurements were done by placing ROI. Two ADC values and two ADC ratios were obtained by different methods. The diagnostic accuracies of these four techniques were compared.

Results: Mean ADC values and ratios of benign and malignant lesions were statistically significant in all of four methods to quantify ADC $(p<0.001)$. Highest positive value and negative predictive value, and diagnostic accuracy rates were achieved when the most restricted part ADC value was calculated. However; highest sensitivity rate and negative predictive value were achieved by calculating the ratio of darkest point ADC to contralateral breast tissue. Positive predictive value, negative predictive value, and diagnostic accuracy rate of calculated ADC values and ratios were higher when lesions were larger than the mean size $\left(3.15 \mathrm{~mm}^{2}\right)$.

Conclusion: Highest diagnostic accuracy rate was obtained with most restricted part ADC value. Obtained ratios by calculating contralateral breast tissue ADC value did not improve the diagnostic accuracy rate. Positive and negative predictive values and diagnostic accuracy rates of ADC values and ratios increased as the lesion size increased.
\end{abstract}

Key Words: Breast mass, Diffusion weighted imaging, $A D C$ value, $A D C$ ratio, Normalised $A D C$.

How to cite this article: Duran B, Ucpinar BA. Four Different Apparent Diffusion Coefficient Measurement Methods in Breast Masses. J Coll Physicians Surg Pak 2021; 31(09):1024-1029.

\section{INTRODUCTION}

Diffusion-weighted imaging (DWI) has been used for the past two decades in the evaluation of breast masses. It was first utilised by Englander et al. and several studies have been published about this evolving technique. ${ }^{1-4}$ These studies stated that DWI has superiority to conventional magnetic resonance imaging (MRI) in the differentiation of benign and malignant breast masses. ${ }^{5}$ DWI is an MRI technique that uses the signal contrast that arises from the differences in Brownian motion of the water molecules. ${ }^{5,6}$ In the human body; water molecules are found in intracellular and extracellularcompartments. As extracellular water molecules freely diffuse, intracellular water molecules have relatively restricted diffusion.

Correspondence to: Dr. Burcin Agridag Ucpinar, Department of Radiology, Sisli Hamidiye Etfal Training and Research Hospital, Istanbul, Turkey

E-mail: drburcinagridag@gmail.com

Received: April 04, 2021; Revised: July 21, 2021;

Accepted: August 20, 2021

DOI: https://doi.org/10.29271/jcpsp.2021.09.1024
DWI gives quantitative and qualitative data about the diffusion properties. ${ }^{6}$ Restriction of water diffusion can be calculated by the apparent diffusion coefficient (ADC). It has been shown that malignant tumors have lower ADC values than benign tumors as they consist of more cell count and intracellular water.

Several studies have been published for the diagnostic value of ADC values in the differentiation of breast tumors. ${ }^{1-3,5}$ A meta-analysis based on 13,847 breast lesions $(10,622$ benign and 3,225 malignant) concluded that an ADC threshold of $1.0 \times 10^{-3}$ $\mathrm{mm}^{2} / \mathrm{s}$ can be used to differentiate benign lesions from breast cancer. ${ }^{5}$ Various methods have been used to obtain ADC values by placing regions of interest (ROI) to the darkest point on the ADC map, to the whole lesion, and to multiple sites of the lesion and calculating the mean value. ${ }^{7-9}$ In the study of Mcdonald et al.; it is stated that the ADC values of normal breast tissue were higher in patients with dense breast tissue on mammography. ${ }^{10}$ However; there is no consensus on ADC measurement method.

Thisstudy was aimed to compare the effect of different measurement methods of apparent diffusion coefficient (ADC) values in the evaluation of breast lesions; and determine its diagnostic value in distinguishing benign/malignant lesions; and evaluate 
the possible effect of contralateral normal breast tissue ADC value by calculating ADC ratios.

\section{METHODOLOGY}

This study was conducted in Sisli Etfal Training and Research Hospital, Turkey, between February 2019 and December 2020. Three hundred and twenty-eight patients, who were admitted to breast radiology clinics for either screening or diagnostic purposes and had breast MRI with DWI between February 2019 and December 2020, were evaluated. Among them, female patients, who had breasttumors and undergone core biopsy, were included in the study. Exclusion criteria were male gender, patients who had biopsy proven bilateral breast cancer; patients who had breast biopsy of surgery history during six months before the MRI scan, patients who received chemotherapy or radiotherapy before the MRI scan, and patients with extensive artifacts in DWI, patients with implants, and patients whose core biopsy, excisional biopsy or postoperative histopathological reports could not be obtained.

This study was conducted in accordance with the 1964 Helsinki Declaration and approved by the Ethics Committees of the Hospital. Since the study was retrospective, informed consent by patients was waived. MRIs were acquired using 1.5 Tesla Siemens scanner (Avanto, Erlengen Germany). In premenopausal women, imaging was performed within 7-14 days of the cycle to prevent possible effects of the menstrual cycle on ADC. Patients were positioned in prone position. Conventional MRI protocol was applied as $\mathrm{T} 1$ weighted fast spin echo axial sequence $(\mathrm{TR}=650, \mathrm{TE}=112$, Matrix $448 \times 224, \mathrm{FOV}=320 \times 320 \mathrm{~mm}, \mathrm{NEX}=1$, Thickness $=3.0$ $\mathrm{mm})$ and pre- and post-contrast T1 weighted three-dimensional fat-suppressed axial sequence ( $T R=485, T E=10$, Matrix $350 \times$ $350, \mathrm{FOV}=320 \times 320 \mathrm{~mm}, \mathrm{NEX}=1, \mathrm{FA}=10.0$, Thickness 3.00). Images were taken before contrast administration and five times after contrast injection with $80 \mathrm{~s}$ intervals. Contrast agent is injected with a dose of $0.1 \mathrm{mmol} \mathrm{kg}$. Diffusion-weighted images $[\mathrm{TR} / \mathrm{TE}=1000 / 83, \mathrm{NEX}=2$ and Thickness $=2 \mathrm{~mm}, \mathrm{FOV}=320 \mathrm{~mm}$, Matrix 180x238] were obtained before contrast and ADC maps were attained.

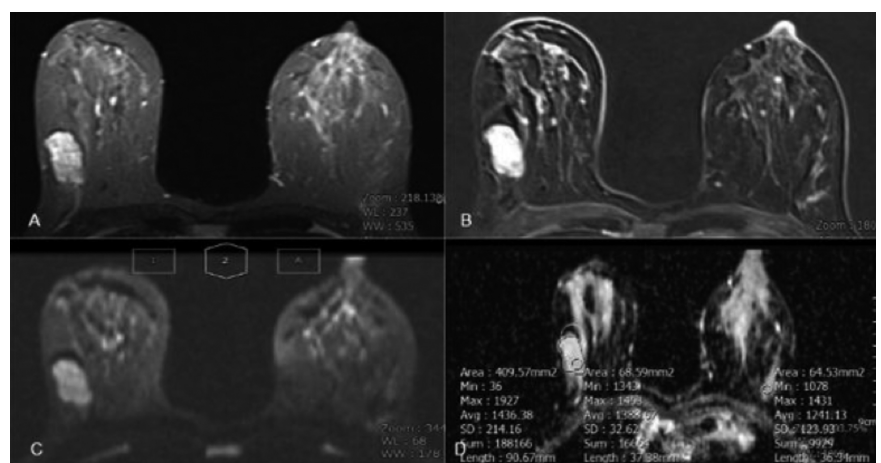

Figure 1: (A) Fat saturated T1-weighted, (B) Subtraction, (C) Diffusion weighted images and (D) ADC map of lobulated homogeneous mass. This lesion was a biopsy proven fibroadenoma. Homogeneous texture of the lesion makes ADC quantifications easier. Three ROI placements are shown here.

All MRIs were evaluated by a radiologist with over eight years of experience in breast MRI interpretation. While placing the ROI,
$\mathrm{T} 1, \mathrm{~T} 2$ and contrast enhanced $\mathrm{T} 1$ imagings were evaluated as reference. ADC mapping was evaluated at the site, which showed highest diffusion restriction, at the site of whole tumor size, and contralateral normal breast tissue. Region of interest (ROI) volume was freely selected rather than using a specific volume. However, the smallest size of ROI placement was carefully selected while placing the ROI to the darkest point on ADC map. Maximum attention was given not to include either the cystic, necrotic, hemorrhagic parts of the tumor or normal breast parenchyma. ADC values were measured by two different methods by placing ROI in the area covering the entire lesion and then in the most restricted area of the lesion on ADC map (Figures 1 and 2). For lesions with homogeneous signal intensity on DWI and ADC map, ROI was placed to the most enhanced part in post contrast T1-weighted images. Three ROI placements were done for each quantification and ROI with the lowest $A D C$ value was selected from multiple ROIs within the targeted area. This ADC value was considered as the minimum $A D C$. Then, three ROIs were placed in the contralateral breast area without lesions, and ADC measurement in the normal breast was made and the average was taken. Subsequently, two ADC values and two ADC ratios were obtained by different methods: 1) from the whole tumor; 2 ) from the most diffusion restricted part on ADC map; 3) ratio of whole tumor ADC value to the contralateral normal breast tissue ADC value; and 4) ratio of the most diffusion restricted part on $A D C$ map to contralateral normal breast tissue ADC value. ADC values were measured from localising a ROI to two dimension ADC maps. The diagnostic accuracies of these four techniques were compared. To interpret DWI, b0 and b1000 sn/ $/ \mathrm{mm}^{2}$ were used. DWIs were obtained before contrast administration to prevent the possible effect of the contrast agent on DWI of the tumor and to prevent $\mathrm{T} 2$ shortening secondary to contrast.

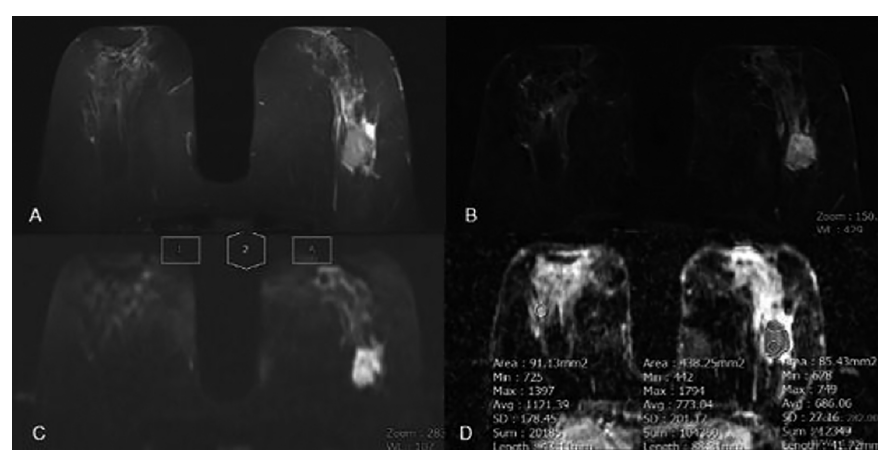

Figure 2: (A) Fat saturated T1-weighted, (B) Subtraction, (C) Diffusion weighted images and (D) ADC map of heterogeneous mass. This lesion was biopsy proven invasive ductal carcinoma. Three ROI placements are shown here. Maximum attention was given to place the ROI to the most restricted (darkest) part of the tumoron ADC map.

SPSS version 15.0 for Windows programme is used for statistical analysis. Descriptive statistics; number and percentage for categorical variables, mean, standard deviation, median and interquartile range for numerical variables (25th and 75th percentile). When comparisons of numerical variables between two independent groups did not provide normal distribution, 
Table I: Comparison of ADC values and ratios achieved by four different ADC quantification methods.

\begin{tabular}{|c|c|c|c|c|c|c|c|}
\hline & ROI & Mean & SD & 25th percentile & 75th percentile & Median & p-value \\
\hline \multirow{2}{*}{ Maximum tumor diameter } & Malignant & 30.4 & 23.9 & 18 & 33 & 25 & \multirow{2}{*}{$<0,001$} \\
\hline & Benign & 22.9 & 20.4 & 12 & 26.75 & 15.5 & \\
\hline \multirow{2}{*}{ Whole lesion ADC $\times 10^{-3} \mathrm{~mm}^{2} / \mathrm{sn}$} & Malignant & 0.96 & 0.19 & 0.85 & 1.06 & 0.95 & \multirow{2}{*}{$<0,001$} \\
\hline & Benign & 1.32 & 0.22 & 1.17 & 1.46 & 1.29 & \\
\hline \multirow{2}{*}{ Darkest point ADC $\times 10^{-3} \mathrm{~mm}^{2} / \mathrm{sn}$} & Malignant & 0.79 & 0.17 & 0.68 & 0.86 & 0.77 & \multirow{2}{*}{$<0,001$} \\
\hline & Benign & 1.21 & 0.23 & 1.05 & 1.37 & 1.21 & \\
\hline \multirow{2}{*}{$\begin{array}{l}\text { Whole lesion/contralateral breast } \\
\text { ADC } \times 10^{-3} \mathrm{~mm}^{2} / \mathrm{sn}\end{array}$} & Malignant & 0.74 & 0.15 & 0.64 & 0.83 & 0.72 & \multirow{2}{*}{$<0,001$} \\
\hline & Benign & 1.02 & 0.20 & 0.91 & 1.09 & 0.98 & \\
\hline \multirow{2}{*}{$\begin{array}{l}\text { Darkest point/contralateral breast } \\
A D C \times 10^{-3} \mathrm{~mm}^{2} / \mathrm{sn}\end{array}$} & Malignant & 0.60 & 0.14 & 0.52 & 0.68 & 0.60 & \multirow{2}{*}{$<0,001$} \\
\hline & Benign & 0.94 & 0.20 & 0.80 & 1.01 & 0.93 & \\
\hline
\end{tabular}

Table II: Predictive values, diagnostic accuracy rates and cut-off values of used four ADC quantification methods.

\begin{tabular}{|c|c|c|c|c|c|c|c|c|c|c|}
\hline & AUC & $95 \% \mathrm{Cl}$ & $\begin{array}{l}\text { Cut-off } \\
\text { value }\end{array}$ & Sensitivity & Specificity & PPV & NPV & $\begin{array}{l}\text { Diagnostic } \\
\text { Accuracy }\end{array}$ & $\begin{array}{l}\text { Kappa } \\
\text { value }\end{array}$ & p-value \\
\hline Whole lesion ADC & 0.896 & $0.855-0.936$ & $\geq 1,100$ & $87.0 \%$ & $81.4 \%$ & $76.3 \%$ & $90.1 \%$ & $83.7 \%$ & 0.669 & 0.038 \\
\hline Most restricted part ADC & 0.932 & $0.899-0.965$ & $\geq 0,936$ & $90.0 \%$ & $87.7 \%$ & $83.3 \%$ & $92.8 \%$ & $88.7 \%$ & 0.767 & 0.185 \\
\hline $\begin{array}{l}\text { Whole lesion/contralateral } \\
\text { breast ADC }\end{array}$ & 0.901 & $0.863-0.938$ & $\geq 0,851$ & $86.0 \%$ & $81.4 \%$ & $76.1 \%$ & $89.4 \%$ & $83.3 \%$ & 0.660 & 0.060 \\
\hline $\begin{array}{l}\text { Most restricted part / } \\
\text { contralateral breast ADC }\end{array}$ & 0.941 & 0.914-0.969 & $\geq 0,709$ & $92.0 \%$ & $82.9 \%$ & $78.6 \%$ & $93.8 \%$ & $86.7 \%$ & 0.729 & 0.005 \\
\hline
\end{tabular}

Mann-Whitney U-test was used. ROC curve analysis was performed for cut-off values. The consistency of the results was examined with the kappa Value. The rates in dependent groups were compared with the McNemar test. Statistical significance level of alpha was accepted as $p<0.05$.

\section{RESULTS}

A total of 246 MRI scans of 246 (100 benign, 146 malignant) breast tumors were finally studied. The mean age of patients was $49.3 \pm 8.7$ years ranging between 21-79 years. According to the pathology results, most common benign lesion was fibroadenoma $(n=68,27.64 \%)$ and most common malignant lesion was invasive ductal carcinoma $(n=104$, $42.28 \%$ ). Core biopsy was performed in 68 fibroadenoma cases, There were eight cases of mastitis and three cases of abscess, on which biopsy was performed due to complex mass-like appearance on ultrasound. Solid parts were carefully biopsied. Mean area of the masses in two dimensions was $3.15 \pm 0.21 \mathrm{~cm}^{2}$ with maximal diameters between 0.6 and $31.6 \mathrm{~cm}$. The mean tumor size of benign and malignant lesions was measured $22.9 \pm 20.4 \mathrm{~mm}$ and $30.4 \pm 23.9 \mathrm{~mm}$, respectively.

Mean ADC values and ratios of benign and malignant lesions were statistically significant in all of four used measurement methods to quantify ADC $(p<0.001)$ with mean ADC values of the malignant lesions were significantly lower than benign ones (Table I). Cut-off values of ADC values and ratios were determined with ROC analysis (Table II). Highest positive and negative predictive value and diagnostic accuracy rate were achieved when the most restricted part ADC value was calculated.

Additionally, diagnostic accuracy of ADC improved when the tumor size in two dimensions was higher than the mean tumor size; as there was a dramatic decrease in positive predictive value and diagnostic accuracy rate of $A D C$ values when the tumor size is under mean tumor size (Table III).

\section{DISCUSSION}

Breast MRI is a widely used diagnostic modality to evaluate the breast with high sensitivity that is reported as $89-100 \%{ }^{1-13}$ MRI has superiority to ultrasonography and mammography as it helps detection of breast lesions in dense breast tissue, identifies the accompanying invasive components. Additionally, breast MRI is the chosen method for screening high risk patients and also assessment of treatment response. ${ }^{4,8,9,11}$ However; the specificity of conventional contrast enhanced MRI is not as high as its sensitivity. Zhang et al. found the sensitivity and specificity of dynamic contrast-enhanced magnetic resonance imaging in determining lesion morphology and kinetics as $93 \%$ and $71 \%$, respectively. At this point, advanced MRI applications have been introduced to identify benign and malignant lesions more accurately.

Englander et al. first utilised DWI in breast tissue in $1997 .{ }^{4}$ In the past two decades; many studies have been published regarding the use of $A D C$ values in further work-up of the 
breast lesions. ${ }^{1-16}$ No need to contrast administration is one of the advantages of DWI. In this regard, DWI may be an alternative for patients in whom contrast agents cannot be administered (patients with severe renal dysfunction and at risk of nephrogenic systemic fibrosis or patients who developed anaphylaxis or severe allergic reaction to gadolinium-based contrast agents. ${ }^{17}$ Additionally, giving quantitative results and short acquisition times (typically 2-4 minutes) are superiorities of DWI and makes it an acceptable imaging method candidate. ${ }^{18}$

Discrepancy in mean ADC values of benign and malignant breast lesions were found in the literature; Guo et al. reporting $1.57+/-0.23 \times 10^{-3} \mathrm{~mm}^{2} / \mathrm{s}, 0.97+/-0.20 \times 10^{-3}$ $\mathrm{mm}^{2} / \mathrm{s}$; Orguc et al. stating $2.00+/-0.55 \times 10^{-3} \mathrm{~mm}^{2} / \mathrm{s}, 1.04$ $+/-0.29 \times 10^{-3} \mathrm{~mm}^{2} / \mathrm{s}$, and Altay et al. describing $1.47+/-$ $0.25 \times 10^{-3} \mathrm{~mm}^{2} / \mathrm{s}, 0.96+/-0.25 \times 10^{-3} \mathrm{~mm}^{2} / \mathrm{s}^{1,14,16}$ The present authors believe that different measures are the result of various size and localisation_of ROI placement and quantification methods as there is no standardisation in ADC measurement. ${ }^{4,8,9,11,19}$ Differences in ADC values demonstrate that radiologists need the results of various quantitative techniques before utilising general cut-off values in clinical practice. For this reason, we aimed to investigate the role of DWI in breast cancer diagnosis by comparing ADC values and ratios that were obtained by three different ROI placements.

In this study, the mean ADC values and ratios quantified by four different measurements were significantly lower in malignant masses; which was concordant with the literature. $^{1-12}$

Many different techniques for ROI placement have been used. Gity et al. placed the ROI to whole lesion and to the most prominent diffusion restricted point of the lesion in DWI. ${ }^{7}$ Aydın et al. placed the ROI to the darkest point of the lesion on ADC map. ${ }^{12}$ Bickel et al. used the minimum, average and maximum $A D C$ values quantified by $2 D$ and $3 D$ ROls. ${ }^{19}$ This study revealed that placing the ROI has an essential role in the performance of DWI and ADC values. ROI placed to the darkest point on ADC map performs better in terms of sensitivity, specificity, positive predictive value, negative predictive value and diagnostic accuracy rate with a cut-off value of $0.936 \times 10^{-3} \mathrm{~mm}^{2} / \mathrm{s}(\mathrm{Cl}=0.899-0.965)$, when compared with ROI placed to the whole lesion on ADC map. However, highest sensitivity rate $(92.0 \%)$ and negative predictive value $(93.8 \%)$ were achieved by calculating the ratio of darkest point ADC to contralateral breast tissue. On the other hand, ratio of the darkest point ADC to contralateral breast ADC has higher sensitivity, specificity, positive predictive value, negative predictive value and diagnostic accuracy rate with a cut-off value of $0.709 \times 10^{-3} \mathrm{~mm}^{2} / \mathrm{s}$; when compared to the ratio of whole lesion ADC to contralateral breast ADC (Table II). The obtained $A D C$ values of benign and malignant breast lesions are exceedingly connected to ROI placement and the size of the ROI. Internal signal heterogeneity of the breast lesions leads to different calculated ADC values and it depends on whether only small areas with relatively homogeneous ADC, larger areas with less homogeneous ADC, or even the whole lesion (especially when necrosis or hemorrhage are present within the lesion) that ROI is placed into.

The ADC values are directly affected by $b$ values selected. ${ }^{5,6}$ In the literature; with the use of higher $b$ values ( $>500$ $\mathrm{mm}^{2} / \mathrm{s}$ ) and using at least two values is necessary to acquire more reliable results. ${ }^{17,19,20}$ There is no evidence to suggest that choice of minimum $b$ value improves diagnostic performance (i.e. 0 or $50 \mathrm{sec} / \mathrm{mm}^{2}$ ). ${ }^{21}$ Variable ADC cut-off values found to discriminate benign and malignant breast lesions so far. In this point; ADC normalisation gains importance as it eliminates the effect of chosen $b$ value as it is a ratio and enables radiologists to perform independent and parallel measurements across institutions. Azab et al. calculated the $A D C$ value by placing multiple ROI in the lesion and selecting the minimum one. Then they divided minimum ADC to contralateral normal breast tissue ADC to achieve normalised ratio. In their study; they found the cut-off value of 0.9 with high sensitivity (92.2\%) and specificity (94.4\%) differentiating benign from malignant. ${ }^{22}$ In this study, the authors performed the same method and found a cut-off value of 0.941 with high sensitivity (92\%) and specificity (82.9\%). When compared, the cut-off value used in this study has significant low specificity with nearly the same sensitivity. In another study which consisted of 93 patients with 101 breast lesions; adding normalised ADCs to contrast enhanced breast MRI improved the diagnostic performance with increase in the area under the curve (AUC) from 0.89 to $0.98 .^{23}$ They calculated multiple ADC values from the lesion and the breast parenchyma and used the median ADC value to calculate the normalized ratio. The present results are controversial in terms of normalised ADC values that AUC and false negative ratio improved. However, the highest diagnostic accuracy rate $(88.7 \%)$ was achieved with the most restricted part ADC value. The difference can be the result of different ADC quantifying methods that the authors used.

In 2019, European Society of Breast Radiology (EUSOBI) international breast DWI working group published a paper to issue a consensus statement in image processing, visualisation and interpretation of DWI in breast MRI. While the group had no consensus on the size of ROI to be used, they suggested to select the minimum ADC value within the Iesion, which was similar to the literature; as it would provide more accurate discrimination between benign and malignant lesions. ${ }^{17}$ These results were consistent with the consensus statement that placing the ROI to the darkest point on ADC map resulted in an increase in sensitivity, specificity, positive predictive value and diagnostic accuracy rate.

In another aspect, the positive predictive value, negative predictive value and diagnostic accuracy rate of calculated $A D C$ values and ratios were higher when lesions were larger 
than the mean size (Table III). ROI placement in smaller lesions constitutes a problem. The authors believe that taking the mass size into account would be a logical approach while evaluating ADC values.

Time spent for ROI placement and ADC measurement is highly important that influences the applicability of this imaging method. In this study, placing the ROls to the large heterogeneous tumors took longer when compared with ROI placement to the smallest restricted area on ADC map. In large tumors, placing ROI to the most restricted part was difficult in tumors with necrotic areas where drawing complex ROIs around the necrotic parts was necessary. In this regard, placing smaller ROIs has the further advantage of shortening the time spent for ADC measurements.

There are several limitations of this study. Firstly, due to its retrospective design, the study results could not be utilised in clinical diagnosis. As a result, this study has a lack of information about different ADC quantification methods on the decision of treatment management. Secondly, patients included in the study were derived from a single clinic. Thirdly, all the ADC calculations were obtained by a single radiologist which resulted in lack of information about interobserver variability. Lastly, the institutional standard protocol was performed to obtain MR images. The calculated ADC value is affected by the scanning parameters (TR and TE), and $b$ value used for DWI. It was believed that there is a need for a consensus approach in terms of MRI parameters.

\section{CONCLUSION}

ADC ratio achieved by division of the whole lesion or darkest point ADC values divided by contralateral breast tissue did not improve the diagnostic accuracy rate, when compared with the diagnostic accuracy of whole lesion ADC value and darkest point ADC value. Highest diagnostic accuracy rate was obtained from the measurement of darkest point $A D C$ value. In addition, positive and negative predictive values and diagnostic accuracy rates of ADC values and ratios increase as the lesion size increases.

\section{ETHICAL APPROVAL:}

This study was conducted in accordance with the 1964 Helsinki Declaration and approved by the Ethics Committee of the Hospital.

\section{PATIENTS' CONSENT:}

The consents of the patients was waived.

\section{CONFLICT OF INTEREST:}

The authors declared no conflict of interest.

\section{AUTHORS' CONTRIBUTION:}

BD, BAU: Contributed to the study conception and design.

BD: Data collection and analysis.

BAU: Wrote first draft of manuscript.
All authors read and approved the final manuscript.

\section{REFERENCES}

1. Guo Y, Cai YQ, Cai ZL, Gao YG, An NY, Ma L, et al. Differentiation of clinically benign and malignant breast lesions using diffusion-weighted imaging. J Magn Reson Imaging 2002; 16(2):172-8. doi: 10.1002/jmri.10140.

2. Sinha S, Lucas-Quesada FA, Sinha U, DeBruhl N, Bassett LW. In vivo diffusion-weighted MRI of the breast: Potential for lesion characterisation. J Magn Reson Imaging 2002; 15(6):693-704. doi: 10.1002/jmri.10116.

3. Kinoshita $T$, Yashiro N, Ihara N, Funatu H, Fakuma E, Narita M. Diffusion-weighted half-Fourier single-shot turbo spin echo imaging in breast tumor: Differentiation of invasive ductal carcinoma from fibroadenoma. I Comput Assist Tomogr 2002; 26(6):1042-6. doi: 10.1097/00004728200211000-00033.

4. Englander SA, Uluğ AM, Brem R, Glickson JD, van Zijl PC. Diffusion imaging of human breast. NMR Biomed 1997; 10(7):348-52. doi: 10.1002/(sici)1099-1492(199710)10: 7<348: aid-nbm487>3.0.co;2-r.

5. Surov A, Meyer HJ, Wienke A. Can apparent diffusion coefficient (ADC) distinguish breast cancer from benign breast findings? A meta-analysis based on 13847 lesions. BMC Cancer 2019; 19(1):955. doi: 10.1186/s12885-019-6201-4.

6. Baliyan V, Das CJ, Sharma R, Gupta AK. Diffusion weighted imaging: Technique and applications. World J Radiol 2016; 8(9): 785-98. doi: 10.4329/wjr.v8.i9.785.

7. Gity M, Moradi B, Arami R, Arabkheradmand A, Kazemi MA. Two different methods of region-of-interest placement for differentiation of benign and malignant breast lesions by apparent diffusion coefficient value. Asian Pac J Cancer Prev 2018; 19(10):2765-70. doi: 10.22034/APJCP.2018. 19.10. 2765.

8. Partridge SC, Rahbar H, Murthy R, Chai X, Kurland BF, DeMartini WB, et al. Improved diagnostic accuracy of breast MRI through combined apparent diffusion coefficients and dynamic contrast-enhanced kinetics. Magn Reson Med 2011; 65(6):1759-67. doi: 10.1002/mrm.22762.

9. Hirano M, Satake H, Ishigaki S, Ikeda M, Kawai H, Naganawa $S$. Diffusion-weighted imaging of breast masses: Comparison of diagnostic performance using various apparent diffusion coefficient parameters. AJR Am J Roentgenol 2012; 198(3):717-22. doi: 10.2214/AJR.11. 7093.

10. McDonald ES, Schopp JG, Peacock S, DeMartini WB, Rahbar $H$, D Lehman C, et al. Diffusion-weighted MRI: Association between patient characteristics and apparent diffusion coefficients of normal breast fibroglandular tissue at $3 \mathrm{~T}$. AJR Am J Roentgenol 2014; 202(5):496-502. doi: 10. 2214/AJR.13.11159.

11. Park MJ, Cha ES, Kang BJ, Ihn YK, Baik JH. The role of diffusion-weighted imaging and the apparent diffusion coefficient (ADC) values for breast tumors. Korean J Radiol 2007; 8(5):390-6. doi: 10.3348/kjr.2007.8.5.390.

12. Aydin H, Guner B, Esen Bostanci I, Bulut ZM, Aribas BK, Dogan $L$, et al. Is there any relationship between adc values of diffusion weighted imaging and the histopathological 
prognostic factors of invasive ductal carcinoma? Br / Radiol 2018; 91(1084):20170705. doi: 10.1259/bjr.20170705.

13. Zhang L, Tang M, Min Z, Lu J, Lei X, Zhang X. Accuracy of combined dynamic contrast-enhanced magnetic resonance imaging and diffusion weighted imaging for breast cancer detection: A meta-analysis. Acta Radiol 2016; 57(6):651-60. doi: 10.1177/0284185115597265.

14. Orguc S, Basara I, Coskun T. Diffusion-weighted MR imaging of the breast: Comparison of apparent diffusion coefficient values of normal breast tissue with benign and malignant breast lesions. Singapore Med J 2012; 53(11):737-43.

15. Woodhams R, Matsunaga K, Iwabuchi K, Kan S, Hata H, Kuranami $M$, Watanabe $M$, Hayakawa K. Diffusion-weighted imaging of malignant breast tumors: The usefulness of apparent diffusion coefficient (ADC) value and ADC map for the detection of malignant breast tumors and evaluation of cancer extension. J Comput Assist Tomogr 2005; 29(5):644-9. doi: 10.1097/01.rct.0000171913.74086.1b.

16. Altay C, Balci P, Altay S, Karasu S, Saydam S, Canada T, et al. Diffusion-weighted MRI: Role in the differential diagnosis of breast lesions. JBR-BTR 2014; 97(4):211-6. doi: 10.5334/ jbr-btr.80.

17. Baltzer $P$, Mann RM, lima $M$, Sigmund EE, Clauser $P$, Gilbert $\mathrm{FJ}$, et al. Diffusion-weighted imaging of the breast - a consensus and mission statement from the EUSOBI international breast diffusion-weighted imaging working group. Eur Radiol 2020; 30(3):1436-50. doi: 10.1007/s00330-01906510-3.
18. Kessler LG, Barnhart HX, Buckler AJ, Choudhury KR, Kondratovich MV, Toledano A, et al. The emerging science of quantitative imaging biomarkers terminology and definitions for scientific studies and regulatory submissions. Stat Methods Med Res 2015; 24(1):9-26. doi: 10.1177/0962280214 537333.

19. Bickel H, Pinker K, Polanec S, Magometschnigg $H$, Wengert G, Spick C, et al. Diffusion-weighted imaging of breast lesions: Region-of-interest placement and different ADC parameters influence apparent diffusion coefficient values. Eur Radiol 2017; 27(5):1883-92. doi: 10.1007/s00330016-4564-3.

20. Koh DM, Collins DJ. Diffusion weighted MRI in the body: Applications and challenges in oncology. AJR Am J Roentgenol 2007; 188(6):1622-35. doi: 10.2214/AJR.06.1403.

21. Baxter GC, Graves MJ, Gilbert FJ, Patterson AJ. A meta-analysis of the diagnostic performance of diffusion MRI for breast lesion characterisation. Radiol 2019; 291(3):632-41. doi: 10.1148/radiol.2019182510.

22. Azab EA, Ibrahim ME. Diffusion weighted (DW) MRI role in characterisation of breast lesions using absolute and normalised ADC values. Egyptian J Radiol Nucl Med 2018; 49:564-70.

23. Ei Khouli RH, Jacobs MA, Mezban SD, Huang P, Kamel IR, Macura $\mathrm{KJ}$, et al. Diffusion-weighted imaging improves the diagnostic accuracy of conventional 3.0-T breast MR Imaging. Radiol 2010; 256(1):64-73. doi: 10.1148/radiol.10091367. 